\title{
Effects of relaxin on the intrauterine distribution and antimesometrial positioning and orientation of rat blastocysts before implantation
}

\author{
P. A. W. Rogers*, C. R. Murphy, K. R. Squires and A. H. MacLennan $\dagger$ \\ Department of Human Morphology, School of Medicine, Flinders University of South Australia, \\ Bedford Park, South Australia 5042, and †Department of Obstetrics and Gynaecology, University of \\ Adelaide, South Australia 5000, Australia
}

\begin{abstract}
Summary. An i.v. infusion of porcine relaxin was administered to rats from the afternoon of Day 4 of pregnancy to the morning of Day 6, a time by which implantation has normally occurred. Implantation sites were irregularly distributed and confined to the cranial half of each uterine horn. Histological sections of uteri fixed by vascular perfusion with osmium tetroxide revealed that the blastocyst was no longer invariably positioned antimesometrially within the lumen and that embryonic disc orientation was often abnormal. A reduced decidual cell reaction was observed around several of the implanting blastocysts.
\end{abstract}

\section{Introduction}

In the rat, implanting blastocysts are distributed more or less evenly along each uterine horn, with implantation always being initiated at the antimesometrial border of the uterine lumen and the embryonic disc within each blastocyst always orientated towards the mesometrium (Finn, 1977). However, the mechanisms responsible for the distribution, positioning and orientation of blastocysts along each uterine horn are poorly understood. Migration of the embryonic disc within the trophoblast has been discounted by Gardner (1975), while other workers have shown that differential uterine growth around each implantation site could be responsible for the more even spacing of such sites (e.g. see Finn, 1968). Rogers, Murphy \& Gannon (1982) demonstrated that such differential uterine movement may also be achieved by tissue hydration, resulting from the increase in endometrial capillary permeability that occurs at each implantation site. McLaren \& Michie (1959) postulated that myometrial activity was responsible for the even distribution of the blastocysts, before implantation, from the utero-tubal junction along each horn. Evidence to support this theory comes from the work of Pusey, Kelly, Bradshaw \& Porter (1980) who used an intravenous infusion of porcine relaxin (a potent inhibitor of myometrial activity in the rat) to disrupt normal distribution before implantation, resulting in the majority of the blastocysts being recovered from the cranial third of each uterine horn. The present work was undertaken as a preliminary study to determine whether the continuation of such a relaxin infusion would result in the majority of implantation sites being similarly confined to the cranial third of each uterine horn, and to examine the effects of relaxin on the normal antimesometrial positioning and orientation of the blastocysts at implantation.

\footnotetext{
* Present address: Department of Obstetrics \& Gynaecology, Queen Victoria Medical Centre, Monash University, Melbourne, Victoria 3000, Australia.
} 


\section{Materials and Methods}

Female Porton rats (262-327 g) showing a pro-oestrous vaginal smear were housed overnight with a male; spermatozoa in the smear the following morning confirmed this day as Day 1 of pregnancy. Each rat was fitted with an indwelling jugular cannula (Silastic tubing, i.d. 0.635 mm, o.d. 1·194 $\mathrm{mm}$; Dow Corning, Midland, Michigan. U.S.A.) under methohexitone anaesthesia $(3.3 \mathrm{ml}$ brietal sodium $/ \mathrm{kg}, 2 \%$ solution in water, i.p.; Eli Lilly (Australia), Sydney) on the morning of Day 3 of pregnancy. The cannula was run s.c. to the top of the head and exteriorized via a dental cement headpiece held in place by 3 watch makers screws fixed into the skull. Rats were housed individually in environmentally controlled cages. The exteriorized cannula (polyethylene tube, i.d. $0.86 \mathrm{~mm}$, o.d. $1.52 \mathrm{~mm}$; Dural Plastics, New South Wales 2158, Australia) was run up to the roof of the cage inside a protective coiled wire spring. At the point where the cannula passed through the roof of the cage a swivel was incorporated in the line to permit it to rotate without kinking. An infusion pump (IM-1, Narashige, Tokyo, Japan), housed outside the cage and calibrated to deliver $0.2 \mathrm{ml} / \mathrm{h}$, was connected to the end of the cannula.

Relaxin was obtained from the ovaries of pregnant sows and was purified by the method of Walsh \& Niall (1980), giving highly purified 'B31' porcine relaxin, which had a biopotency of 1500 GPU/mg relative to the reference preparation NIH-R-P1 (potency $442 \mathrm{GPU} / \mathrm{mg}$ ) (Steinetz et al., 1960).

Two rats were treated as controls, receiving a saline $(9 \mathrm{~g} \mathrm{NaCl} / \mathrm{l})$ infusion from $17: 00 \mathrm{~h}$ on Day 4 of pregnancy to $10: 00 \mathrm{~h}$ on Day 6. Environmentally controlled cages were set for onset of light at $07: 30 \mathrm{~h}$ and onset of darkness at $19: 30 \mathrm{~h}$. Six rats received relaxin infusions $(6.66 \mu \mathrm{g} / \mathrm{h})$ in saline over the same time period.

At $15 \mathrm{~min}$ before autopsy, the rats were given an i.v. injection of $1 \%$ Evans blue in saline, via the indwelling jugular cannula, to permit identification of implantation sites (see Rogers et al., 1982). As soon as the relaxin infusion was completed rats were anaesthetized with i.v. pentobarbitone sodium (40 mg Nembutal $/ \mathrm{kg}$ : Abbott, Sydney), the descending thoracic aorta was rapidly cannulated, and the renal arteries were approached through separate lateral abdominal incisions and clamped shut with haemostats at points close to each kidney. Osmium tetroxide $(50 \mathrm{ml} \mathrm{of} 1 \%$ aqueous solution) in $0 \cdot 1 \mathrm{~m}$-phosphate buffer, $\mathrm{pH} 7 \cdot 4$, containing $9 \mathrm{~g} \mathrm{NaCl} / 1$ was infused via the thoracic cannula, the first $40 \mathrm{ml}$ with a driving pressure of $150 \mathrm{mmHg}$ and the last $10 \mathrm{ml}$ at 50 $\mathrm{mmHg}$. The primary osmium fixation took about $10 \mathrm{~min}$. This was followed by vascular washout of the osmium fixative with $50 \mathrm{ml} 0 \cdot 1 \mathrm{M}$-phosphate buffer, $\mathrm{pH} 7 \cdot 4$, containing $9 \mathrm{~g} \mathrm{NaCl} / 1$ at $100 \mathrm{mmHg}$ driving pressure, and finally with $50 \mathrm{ml} 2.5 \%$ glutaraldehyde in $0 \cdot 1 \mathrm{M}$-phosphate buffer, $\mathrm{pH} 7 \cdot 4$, containing $9 \mathrm{~g} \mathrm{NaCl} / 1$ at $100 \mathrm{mmHg}$. The whole uterus was then carefully excised with a minimum of physical twisting or bending, and further fixed by immersion in $2.5 \%$ glutaraldehyde for $1 \mathrm{~h}$. The whole uterus was rinsed and then stored in $0.1 \mathrm{M}$-phosphate buffer, $\mathrm{pH} 7 \cdot 4$, before being processed for routine light microscope histology.

\section{Results}

Implantation sites in the control rats were evenly distributed along the full length of each uterine horn (Pl. 1, Fig. 1). However, in the rats that had received a relaxin infusion the implantation sites (as indicated by the bands of blue uterine tissue) were always confined to the cranial half of each uterine horn (Pl. 1, Fig. 2). The number of sites per horn ranged from 2 to 6 (mean 4.5 ) and their distribution was variable, with blue bands appearing from almost continuous to well separated. In one instance, 2 implanting blastocysts were found within $120 \mu \mathrm{m}$ of each other, with the primary decidual reactions being continuous.

A histological section of a normal rat implantation site from the morning of Day 6 of pregnancy, showing the antimesometrial positioning of the blastocyst, and the surrounding primary decidual 


\section{PLATE 1}
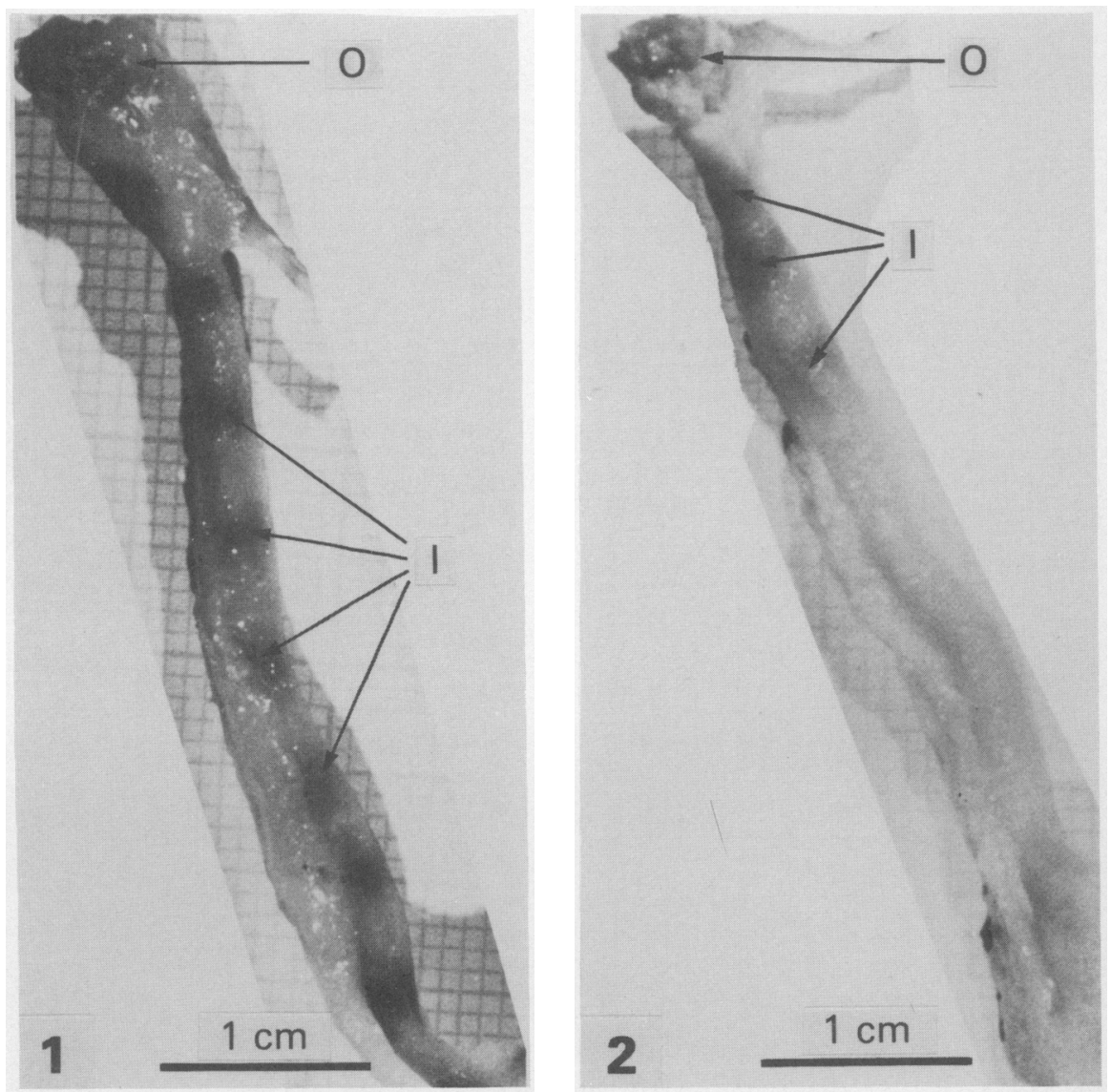

Fig. 1. Uterine horn from a control rat killed on the morning of Day 6 of pregnancy $15 \mathrm{~min}$ after receiving an i.v. injection of Evans blue. Note the relatively even spacing of the implantation sites (I) (represented by the dark bands where Evans blue bound to serum albumin has accumulated in the tissues due to the locally increased endometrial vascular permeability around each implantation site) along the full length of the uterine horn. $O$, ovary.

Fig. 2. Uterine horn from a rat killed on the morning of Day 6 of pregnancy after receiving a continuous infusion of porcine relaxin from the afternoon of Day 4 until the time of death. Note how the implantation sites (I) are irregularly spaced and confined to the cranial half of the uterine horn. $\mathrm{O}$, ovary. 

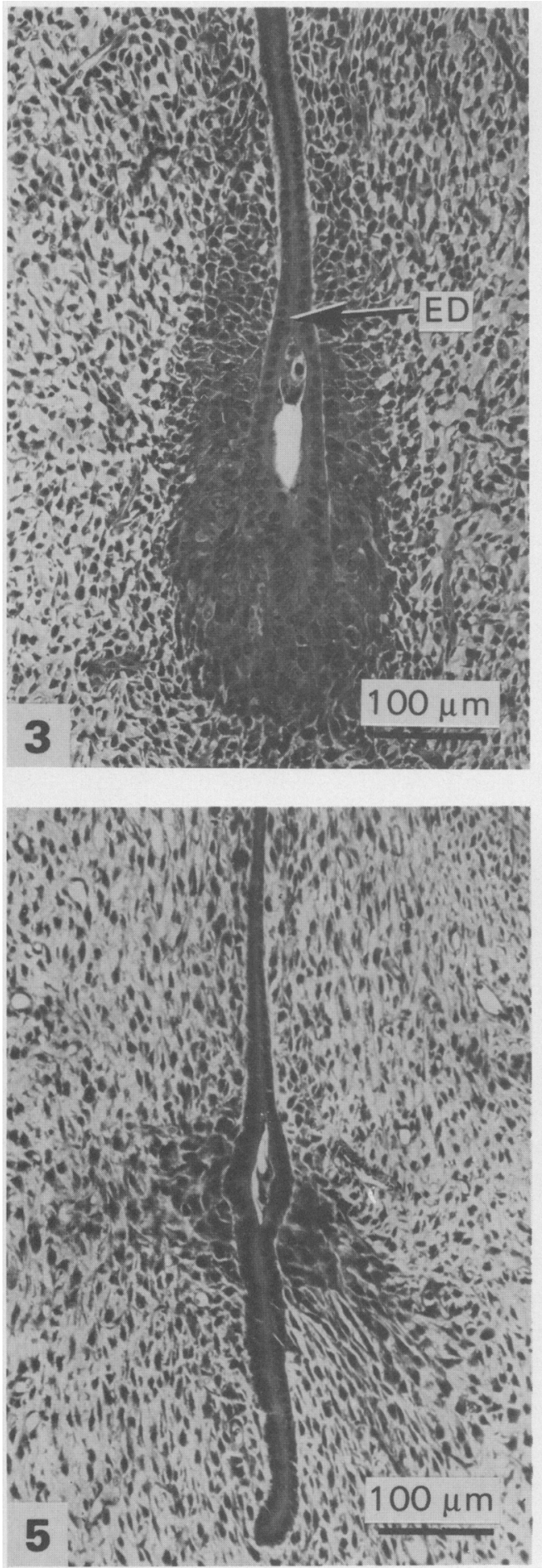
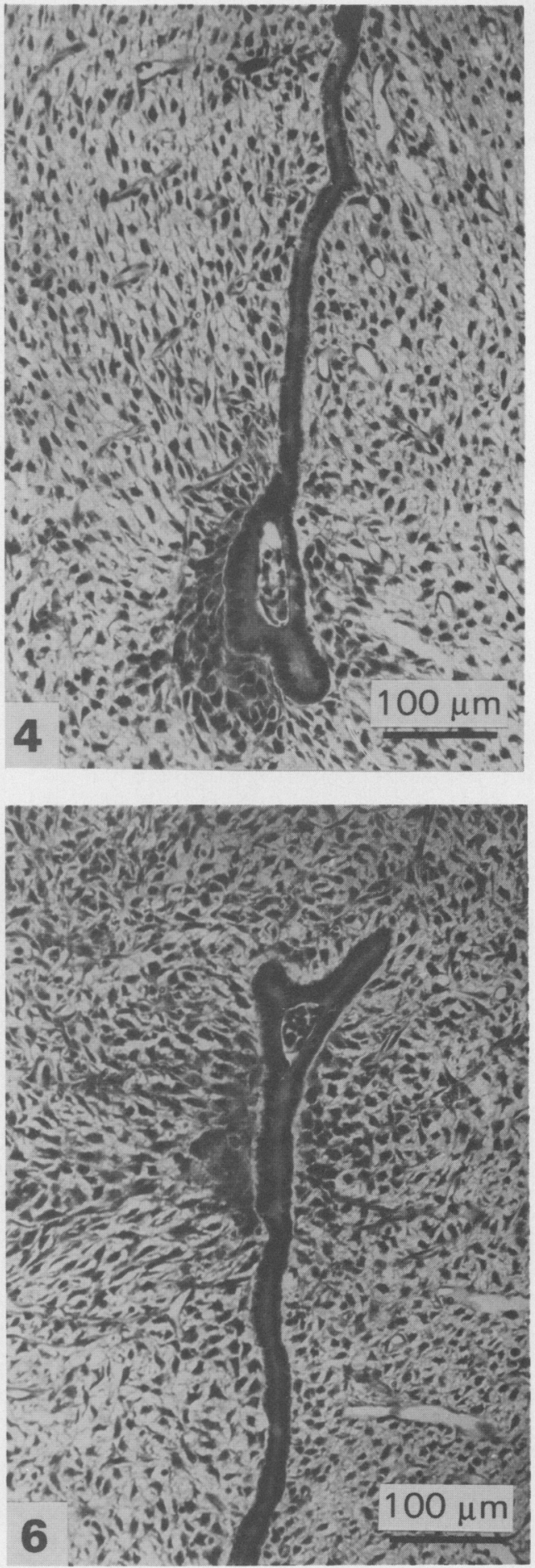
reaction, is illustrated in $\mathrm{Pl}$. 2, Fig. 3. By comparison, examples of implantation sites taken at the same stage of pregnancy, but after an i.v. infusion of relaxin, are shown in Pl. 2, Figs 4-6. The details of blastocyst position and orientation from relaxin treated rats are summarized in Table 1 . Two of the treated rats could not be included in the experimental data because the uterine lumen opened during fixation or histological processing, dislodging the blastocysts, and thus making it impossible to assess their luminal position or orientation accurately.

Table 1. Data for blastocyst positioning and orientation relative to the antimesometrial (A-M) border of the uterine lumen on Day 6 of pregnancy in 4 rats treated with relaxin

\begin{tabular}{|c|c|c|c|}
\hline $\begin{array}{l}\text { No. of } \\
\text { implanting } \\
\text { blastocysts }\end{array}$ & $\begin{array}{l}\text { Distance of } \\
\text { implantation site } \\
\text { from the A-M } \\
\text { border }(\mu \mathrm{m})\end{array}$ & $\begin{array}{c}\text { Distance from A-M } \\
\text { border, as } \% \\
\text { of the total distance } \\
\text { between the A-M } \\
\text { and the mesometrial } \\
\text { luminal borders }\end{array}$ & $\begin{array}{l}\text { No. of blastocysts } \\
\text { in each group showing } \\
\text { non-mesometrial } \\
\text { orientation of the } \\
\text { embryonic disc }\end{array}$ \\
\hline 11 & $0-100$ & $0-9 \%$ & 5 \\
\hline 3 & $101-200$ & $10-13 \%$ & 0 \\
\hline 1 & 260 & $21 \%$ & 1 \\
\hline 1 & 700 & $44 \%$ & 1 \\
\hline 1 & 930 & $89 \%$ & 1 \\
\hline 1 & 1080 & $62 \%$ & 1 \\
\hline
\end{tabular}

Despite the apparently normal positioning of 11 of the blastocysts near the antimesometrial border of the lumen, 5 of these 11 showed abnormal embryonic disc orientation (see Pl. 2, Fig. 4). In most of these 5 cases the embryonic disc was laterally rather than mesometrially directed, although in at least one blastocyst the disc was orientated fully towards the antimesometrial border. The three blastocysts that had implanted 101-200 $\mu \mathrm{m}$ from the antimesometrial border each showed normal embryonic disc orientation. The remaining 4 blastocysts had abnormal embryonic disc orientation.

The extent of the decidual reaction appeared markedly reduced in many of the implantation sites from relaxin-treated rats compared with controls (e.g. compare Pl. 2, Fig. 3 with Pl. 2, Figs 46). However, the degree of decidualization shows large variability even in normal circumstances, and so, due to the limited number of specimens available, quantitative studies of the size or extent of the decidual reaction in relaxin-treated rats were not undertaken in this study.

\section{PLATE 2}

Fig. 3. Histological section of a control implantation site taken on the morning of Day 6 of pregnancy. The blastocyst is positioned antimesometrially within the uterine lumen with the embryonic disc (ED) orientated towards the mesometrium, while the surrounding stromal cells exhibit an early decidual reaction. H \& E. $6 \mu \mathrm{m}$.

Figs 4-6. Histological sections of implantation sites from relaxin treated rats on Day 6 of pregnancy. The antimesometrial margin is at the bottom in all photographs. In each example the embryonic disc is not orientated towards the mesometrium, and in Figs $5 \& 6$ the blastocyst is not positioned at the antimesometrial border of the lumen. Note the reduced decidual reactions compared with that in Fig. 3, and the differences in epithelial thickness on the mesometrial compared with the antimesometrial side of the blastocyst in Fig. 5 . H \& E, $6 \mu \mathrm{m}$. 
In several of the implantation sites located up to $260 \mu \mathrm{m}$ from the antimesometrial border, the luminal epithelium mesometrial to the blastocyst appeared different from that on the antimesometrial side (Pl. 2, Fig. 5); the mesometrially located epithelium was thinner than that on the antimesometrial side of the blastocyst, with individual cells appearing cuboidal rather than columnar. No measurements of epithelial thickness were taken in this study due to the limited numbers of specimens available.

\section{Discussion}

This work demonstrates that blastocysts are able to initiate implantation successfully under the influence of exogenous relaxin, and confirms that the distribution of the implantation sites on Day 6 of pregnancy is similar to that of the preimplantation blastocysts under the influence of relaxin on Day 5 of pregnancy, i.e. restricted to the cranial portion of each uterine horn. It also demonstrates that, under the influence of relaxin, rat blastocysts do not invariably implant at the antimesometrial border of the uterine lumen, and also that the embryonic disc is no longer always orientated towards the mesometrium. To our knowledge this is the first report of non-antimesometrial implantation and/or unusual embryonic disc orientation in the rat, and the technique would therefore be useful to investigate further these complex and poorly understood phenomena.

That 11 of the 18 blastocysts sectioned from relaxin-treated rats were positioned at or near the antimesometrial border of the lumen suggests that the normal antimesometrial positioning mechanism may still have been at least partly effective. This may be due to the relaxin dose being only marginally effective or a more indirect effect. It is possible, for example, that the unusual crowding of all the blastocysts at the ovarian end of the uterine horn due to the effects of the relaxin on the myometrium (Pusey et al., 1980), may disrupt the subsequent antimesometrial positioning mechanism. Of the 11 blastocysts positioned antimesometrially, 5 had unusual embryonic disc orientation, suggesting that the mechanism responsible for orientation may be, in part at least, different from that responsible for antimesometrial positioning. This would also explain the observation that 3 of the blastocysts that did not achieve normal antimesometrial positioning did, however, show normal embryonic disc orientation. A minimum of three separate mechanisms may therefore be involved in normal blastocyst movement before implantation; the first in spacing the blastocysts along the uterine horn, which involves the myometrium, the second in moving the blastocysts to the antimesometrial border, and the third in orientating the blastocysts so that the embryonic disc is towards the mesometrium.

The 4 blastocysts that implanted a substantial distance from the antimesometrial border all showed unusual embryonic disc orientation. Despite this, these sites demonstrate that it is possible for implantation to be initiated well away from the antimesometrial border, and that it is the blastocyst, and not the antimesometrial portion of the endometrium, which is responsible for initiating decidualization.

The differences in epithelial thickness mesometrial to the blastocyst compared with antimesometrially, as seen in some implantation sites (PI. 2, Fig. 5), may be an indication of one of the mechanisms responsible for blastocyst movement. It is possible that these morphological differences seen histologically represent epithelial cell migration in the living tissue. Thus the epithelium may be involved in moving the blastocyst towards its 'correct' position, even though it has already implanted, by migrating towards the antimesometrial border.

We thank Dr John Willoughby for use of the environmentally controlled cages, Peta Jervois for demonstrating the indwelling cannula operation, Ashley Hudson for constructing the cannula line swivel, Dr Bren Gannon for constructive criticism of the manuscript and Jane Deer and Debbie Thompson for typing. The relaxin was purified with the support of a grant from the National Health and Medical Research Council of Australia. 


\section{References}

Finn, C.A. (1968) Increase in length of the uterus at the time of implantation in the mouse. J. Reprod. Fert. 17, 69-74.

Finn, C.A. (1977) The implantation reaction. In Biology of the Uterus, pp. 245-308. Ed. R. M. Wynn. Plenum Press, New York.

Gardner, R.L. (1975) Analysis of determination and differentiation in the early mammalian embryo using intra- and interspecific chimeras. In The Developmental Biology of Reproduction, pp. 207-236. Eds C. L. Markert \& J. Papaconstantinou. Academic Press, New York.

Gil, J. (1980) Organisation of microcirculation in the lung. Ann. Rev. Physiol. 42, 177-186.

McLaren, A. \& Michie, D. (1959) The spacing of implantations in the mouse uterus. Mem. Soc. Endocr. 6, 65-75.
Pusey, J., Kelly, W.A., Bradshaw, J.M.C. \& Porter, D.G. (1980) Myometrial activity and the distribution of the blastocysts in the uterus of the rat: interference by relaxin. Biol. Reprod. 23, 394-397.

Rogers, P.A.W., Murphy, C.R. \& Gannon, B.J. (1982) Changes in the spatial organization of the uterine vasculature during implantation in the rat. $J$. Reprod. Fert. 65, 211-214.

Steinetz, B.G., Beach, V.L., Kroc, R.L., Stasilli, N.R., Nussbaum, R.E., Nemith, P.J. \& Dun, R.K. (1960) Bioassay of relaxin using a reference standard: a simple and reliable method utilizing direct measurement of interpubic ligament formation in mice. Endocrinology 67, 102-115.

Walsh, J.R. \& Niall, H.D. (1980) Use of an octadecylsilica purification method minimizes proteolysis during isolation of porcine and rat relaxins. Endocrinology 107, 1258-1260.

Received 14 December 1982 